\title{
NUCLEAR ATTENUATION OF HADRONS AT HERMES
}

\author{
GAREGIN ELBAKIAN \\ FOR THE HERMES COLLABORATION \\ Yerevan Physics Institute, \\ Yerevan 375036, Armenia \\ elbakian@hermes.desy.de
}

\begin{abstract}
The influence of the nuclear environment on the hadronization of charged pions, kaons and (anti)protons in semi-inclusive deep inelastic scattering has been studied by the HERMES experiment at DESY using a $27.5 \mathrm{GeV}$ positron beam. Identified hadron multiplicities have been measured for helium, neon and krypton relative to thate of deuterium as a function of $\nu, z$ and $p_{T}^{2}$. A large increase in the multiplicity ratios with $p_{T}^{2}$ was observed, clarifying the role of FSI in the nuclear transverse momentum broadening. In addition, double-hadron production in the nuclear medium was investigated. Nuclear effects on the double hadron-production ratio provided an additional tool for studying modifications of hadronization in nuclear matter.
\end{abstract}

Keywords: Hadronization; nuclear broadening; double-hadron attenuation.

\section{Introduction}

The study of hadron production in deep inelastic scattering (DIS) offers the possibility to investigate quark propagation and hadronization. Quark propagation in the nuclear medium involves competing process such as hadronization of quarks, quark energy loss through multiple scattering, and gluon radiation. The relatively clean nuclear environment of lepton-induced reactions allows the investigation of the space-time evolution of the hadronization process already in the early stage of hadron production.

\section{Identified Hadron Attenuation}

The experimental results are presented in terms of the multiplicity ratio $R_{A}^{h}$. This is the ratio of the number of hadrons produced per DIS event for a nuclear target with mass number A to that from a deuterium (D) target. It is defined as

$$
R_{A}^{h}\left(\nu, z, Q^{2}, p_{T}^{2}\right)=\left(\frac{N_{h}^{S I D I S}\left(\nu, Q^{2}, z, p_{T}^{2}\right)}{N_{e}^{D I S}\left(\nu, Q^{2}\right)}\right)_{A} /\left(\frac{N_{h}^{S I D I S}\left(\nu, Q^{2}, z, p_{T}^{2}\right)}{N_{e}^{D I S}\left(\nu, Q^{2}\right)}\right)_{D},
$$

where $z, Q^{2}$ and $p_{T}^{2}$ represent the fraction of the virtual photon energy $\nu$ transferred to the hadron, the virtuality of the photon, and the square of the hadron momentum component transverse to the virtual photon direction, respectively. $N_{h}^{S I D I S}\left(\nu, \mathrm{z}, p_{T}^{2}\right)$ 

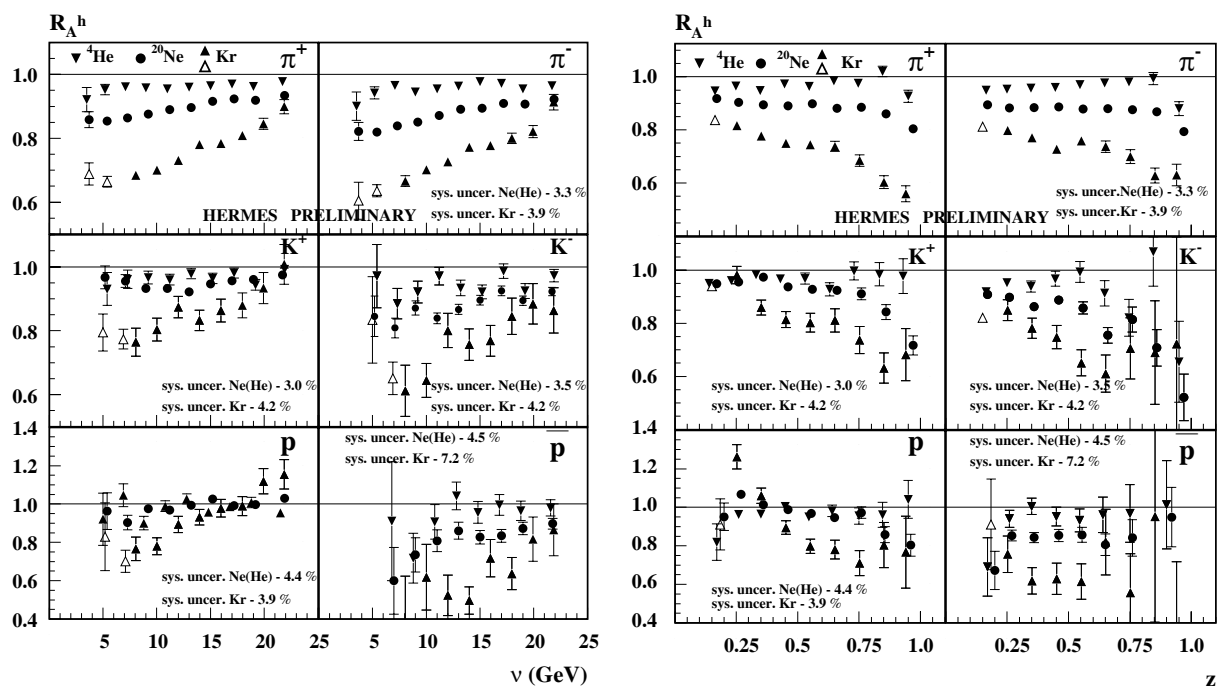

Fig. 1. Hadron multiplicity ratios $R_{A}^{h}$ for $\pi^{ \pm}, K^{ \pm}, p$ and $\bar{p}$ as a function of $\nu$ (left) and $z$ (right) for different nuclei. For the $\mathrm{Kr}$ data the closed triangles are taken from Ref. 2 and the open triangles depict the present results in an extended kinematic range.

is the number of semi-inclusive (SIDIS) hadrons (pions, kaons or (anti)protons) in a given $\left(\nu, \mathrm{z}, p_{T}^{2}\right)$-bin, and $N_{e}^{D I S}\left(\nu, Q^{2}\right)$ the number of inclusive DIS positrons in the same $\left(\nu, Q^{2}\right)$-bin. The multiplicity ratio was determined as a function of $\nu, \mathrm{z}$ and $p_{T}^{2}$, while integrating over all other kinematic variables. Nuclear attenuation is defined as $1-R_{A}^{h}$.

The multiplicity ratio $R_{A}^{h}$ has been measured at HERMES using D, ${ }^{4} \mathrm{He},{ }^{20} \mathrm{Ne}$ and $\mathrm{Kr}$ gas targets internal to the $27.5 \mathrm{GeV}$ HERA positron storage ring, identifying both the scattered positron and the produced hadrons in the HERMES spectrometer ${ }^{1}$.

In Fig. 1 the multiplicity ratio $R_{A}^{h}$ is shown for charged pions, kaons and (anti)protons on ${ }^{4} \mathrm{He},{ }^{20} \mathrm{Ne}$ and $\mathrm{Kr}$ targets as a function of $\nu$ (left panels) and as a function of $z$ (right panels). The data show that $R_{A}^{h}$ is reduced at low $\nu$ and high $z$. The reduction is stronger for heavy nuclei. This clearly shows the influence of the nuclear environment on hadron formation. While $R_{A}^{h}$ for $\pi^{+}$and $\pi^{-}$mesons is similar, a significant difference for the multiplicity ratios is found between $K^{+}$ and $K^{-}$, and for protons and antiprotons. The difference for the kaons may be due to differences in the hadron-nucleon interaction cross section $\left(\sigma_{h N}\right)$ for $K^{+}$and $K^{-}$.

The multiplicity ratio $R_{A}^{h}$ as a function of $p_{T}^{2}$ is shown for $\pi^{ \pm}, K^{ \pm}, p$ and $\bar{p}$ in Fig. 2, left panels. An enhancement of the number of hadrons at high $p_{T}^{2}$ with atomic mass number $A$ is observed. This enhancement is also called the Cronin effect and has previously been reported for proton-nucleus and nucleus-nucleus collisions ${ }^{3}$, but is smaller in magnitude. In contrast to the previous reactions the Cronin effect at 



Fig. 2. Left: multiplicity ratio vs $p_{T}^{2}$ for $\pi^{ \pm}, K^{ \pm}, p$ and $\bar{p}$ on ${ }^{4} \mathrm{He},{ }^{20} \mathrm{Ne}$ and $\mathrm{Kr}$ targets. Right: The ratio $R_{2 h}$ as function of $z_{2}$ with $z_{1}>0.5$. In the upper panel the curves (solid for ${ }^{14} \mathrm{~N}$, dashed for $\mathrm{Kr}$, dotted for $\mathrm{Xe}$ ) are calculated within a BUU transport model ${ }^{7}$. In the bottom panel the same data are shown with calculations that assume only absorption for the three nuclei (same line types as in the upper plot $)^{7}$.

HERMES can only be due to final state interactions. It is further observed that the $p_{T}^{2}$ spectra are determined mainly by the type and charge of the hadrons, and that they depend only weakly on the target mass. Thus, the observed enhancements in the pion and kaon spectra are more suppressed than in the proton spectra.

The HERMES data may help to interpret the relativistic heavy-ion results from $\mathrm{SPS}^{4}$ and $\mathrm{RHIC}^{5}$, which show a weaker $p_{T}$ enhancement than expected from the original Cronin effect.

\section{Double-Hadron Attenuation}

Double-hadron leptoproduction offers an additional tool to study hadronization. The experimental results for $N, K r$ and $X e$ relative to D are presented in terms of the ratio

$$
R_{2 h}\left(z_{2}\right)=\left(\frac{d N^{z_{1}>0.5}\left(z_{2}\right) / d z_{2}}{N^{z_{1}}}\right)_{A} /\left(\frac{d N^{z_{1}>0.5}\left(z_{2}\right) / d z_{2}}{N^{z_{1}}}\right)_{D},
$$

where $z_{1}$ and $z_{2}$ correspond to the leading (largest $z$ ) and sub-leading (second largest $z$ ) hadrons, respectively, $d N^{z_{1}>0.5}$ is the number of events with at least two detected hadrons in a bin of width $d z_{2}$ at $z_{2}$ with $z_{1}>0.5$, and $N^{z_{1}>0.5}$ is the number of events with at least one detected hadron with $z_{1}>0.5$. All charged hadrons and neutral pions are considered. The results on $R_{2 h}$ are generally below unity, and show essentially no A dependence. The HERMES data ${ }^{6}$ clearly show that nuclear 
effects in the double-hadron ratio are smaller than for single-hadron attenuation measured under the same kinematic condition. Fig. 2, right-upper panel, shows calculations by means of a BUU transport model ${ }^{7}$. Although the general trend of the data is reproduced, the model prediction is not supported by the data. Fig. 2, right-bottom panel, shows the same data compared to a calculation with a purely absorptive treatment of the interaction of the pre-hadronic or the final hadronic states. This assumption ${ }^{7}$ is ruled out by the data.

Thus, the double-hadron observables in SIDIS provide, as did the jet correlation measurements in heavy-ion collisions, new information for differentiating between the models of hadronization in nuclei that are indistinguishable in single-hadron measurements.

\section{Acknowledgments}

I am grateful to N. Akopov, D. Hasch and P. di Nezza for useful discussions and comments.

\section{References}

1. K. Ackerstaff et al. [HERMES Collaboration], Nucl. Instr. and Meth. A 417, 230 (1998).

2. A. Airapetian et al., Eur. Phys. J. C 20, 479 (2001).

3. M. Aggarwal et al. [WA98 Collaboration], Phys. Rev. Lett. 81, 4087 (1991) and ibid. 84, 578(E) (2000);

H. Appelshauser et al. [NA49 Collaboration], Phys. Rev. Lett. 82, 2471 (1999).

4. M. Aggarwal et al. [WA98 Collaboration], Eur. Phys. J. C 23, 225 (2002).

5. K. Adcox et al. [PHENIX Collaboration], Psys. Rev. Lett. 88, 022301 (2002).

6. A. Airapetian et al., Phys. Rev. Lett. 96, 162301 (2001).

7. T. Falter et al., Phys. Lett. B 594, 61 (2004); Phys. Rev. C 70, 054609 (2004);

K. Gallmeister, W. Cassing, Nucl. Phys. A 748, 241 (2005). 